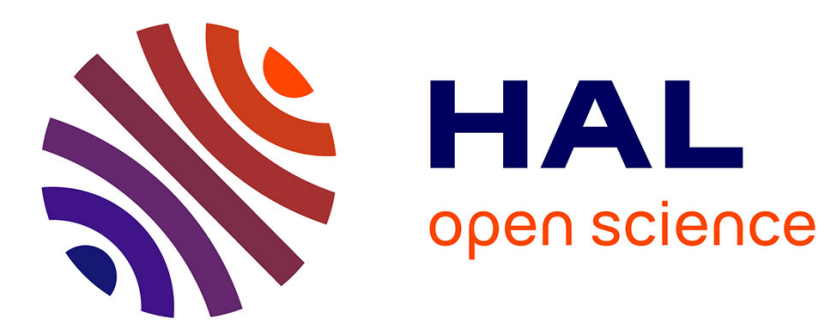

\title{
Bayesian Networks and Evidence Theory to Model Complex Systems Reliability
}

Christophe Simon, Philippe Weber, Eric Levrat

\section{To cite this version:}

Christophe Simon, Philippe Weber, Eric Levrat. Bayesian Networks and Evidence Theory to Model Complex Systems Reliability. Journal of Computers (JCP), 2007, 2 (1), pp.33-43. hal-00139491

\section{HAL Id: hal-00139491 \\ https://hal.science/hal-00139491}

Submitted on 31 Mar 2007

HAL is a multi-disciplinary open access archive for the deposit and dissemination of scientific research documents, whether they are published or not. The documents may come from teaching and research institutions in France or abroad, or from public or private research centers.
L'archive ouverte pluridisciplinaire HAL, est destinée au dépôt et à la diffusion de documents scientifiques de niveau recherche, publiés ou non, émanant des établissements d'enseignement et de recherche français ou étrangers, des laboratoires publics ou privés. 


\title{
Bayesian Networks and Evidence Theory to Model Complex Systems Reliability
}

\author{
Ch. SIMON, Ph. WEBER, E. LEVRAT \\ Centre de Recherche en Automatique de Nancy (CRAN - UMR 7039), Nancy University, CNRS, \\ 2 Rue Jean Lamour, 54509 Vandoeuvre, France \\ Email: \{christophe.simon, philippe.weber, eric.levrat\}@cran.uhp-nancy.fr
}

\begin{abstract}
This paper deals with the use of Bayesian Networks to compute system reliability of complex systems under epistemic uncertainty. In the context of incompleteness of reliability data and inconsistencies between the reliability model and the system modeled, the evidence theory is more suitable to manage this epistemic uncertainty. We propose to adapt the Bayesian Network model of reliability in order to integrate the evidence theory and then to produce an Evidential Network. Three examples are proposed to observe the propagation mechanism of the uncertainty through the network and its influence on the system reliability.
\end{abstract}

Index Terms-Reliability, Epistemic Uncertainty, Dempster Shafer Theory, Bayesian Networks, Evidential Networks

\section{INTRODUCTION}

The studies of reliability interest many fields like chemistry, civil engineering [1], [2], manufacturing processes, transport [3], [4]... Taking into consideration failures and accidents met, this science is of a major interest for companies. In addition, our technological capacities increase and we build systems increasingly complex and large like the nuclear or thermal plants, infrastructure of water distribution or electricity. The reliability studies of such systems are consequently more difficult to carry out.

If current international standards like the IEC61508 [5], IEC61511 [6] or the ISA S96 [7] recommend the use of modeling tools referred like Markov chains, fault trees or the simplified equations, they leave the place to the use of other tools. Markov Chains [8] allow an exact computation of system reliability so complex it is. However, large systems become difficult to model by Markov chains because they induce a combinatory explosion of the states. Fault trees [6], [9] are also difficult to implement on large systems and particularly if the studied system presents redundant failures and where the minimal cuts can not be reduced [10]. In addition, standard fault trees cannot take into account common causes. Lastly, the simplified equations are not exploitable if the reliability engineer is not able to gradually approach the modeling of large complex systems.

As mentioned above, the standard tools presents some difficulties in their implementation for complex or large systems. This is why the reliability engineers and the researchers in the field of reliability sought tools offering more important capacity of modeling. Within this framework, there are improved versions of fault trees, reliability diagrams, switching Markov chains... The Petri nets were also used to model reliability and are very interesting when they are associated with Monte Carlo simulations [11]. However, these improvements make the models more complicated to use.

In this context, the Bayesian Networks are a very interesting tool. They allow the stochastic modeling of reliability in a compact and graphic form. It is possible to logically explain the functional propagation of failures, to introduce the factors of common causes. The stochastic dependencies between events can also be materialized simply. Moreover, it is possible to use the Bayesian Networks from the causal point of view to observe the effect of a failure, to analyze the probable state of some components knowing that of subsystems including them in a diagnostic approach. The Bayesian Networks are thus general-purpose tools making it possible to model knowledge on the reliability of a system whatever its complexity is. In addition, it can be used to manage maintenance actions.

In spite of this high capacity of modeling offered by the Bayesian Networks, the reliability engineer or the engineer in maintenance is not always able to provide an exact model of the studied system. Moreover, he does not have at every moment the data necessary on the state of each component. Indeed, this situation is found when it is necessary to inspect the components when the system is operating. We are thus in the presence of missing data or of potential inconsistency between the data and the model. The inconsistency and the incompleteness point out a problem of uncertainty.

The problem arising from uncertainty is largely tackled in the literature. Indeed, the probabilistic framework was formalized for its treatment. However, it is now well known that uncertainty presents several forms. The random point of view of uncertainty, viewed by frequentist or subjectivist, can be easily taken into account by the Bayesian Networks. The possibility theory also offers a framework to the treatment of uncertainty. The epistemic point of view can not be handled appropriately by the probability theory. The evidence theory finds its interest in this context [12].

Indeed, the rigorous framework of probability on which the Bayesian Networks are based does not make it possible to treat epistemic uncertainty suitably. Ellsberg [13] illustrated this problem using a very traditional problem of ballot box where the knowledge of the number of colored 
balls is not complete. Ellsberg showed that the theory of probability forces to define the probability of pulling of the unnumbered balls in order to respect the theorem of additivity. The definition of this number is then made in the minimal commitment principle which results in a uniform distribution.

The evidence theory or belief function theory brings an interesting solution to the above problem while making it possible to engage no more knowledge than we have in the reasoning [14]. Its formal framework was proposed by Dempster and Shafer in 1976. Many developments have followed. Shenoy [15] developed the Sequential Valuable Networks, a graphical model of knowledge formalization. Smets [14] also proposed his graphical model but based on the formal framework of the Transferable Belief Model.

To model reliability in the epistemic context of uncertainty, the combination of the evidence theory and the Bayesian Networks offers a very interesting tool. In this article, we show how this combination is carried out in the goal of modeling system reliability. For that, Section II is devoted to the basics of Bayesian Network for the reliability modeling. Section III is dedicated to the description of the useful bases of the evidence theory to develop Evidential Networks for reliability modeling. The last section is dedicated to some applications in order to highlight the interest of our proposal.

\section{BAYESIAN NETWORK FORMALISM}

Bayesian Networks are Directed Acyclic Graphs used to represent uncertain knowledge in Artificial Intelligence [16]. A Bayesian Network is defined as a couple: $G=$ $((N, A), D)$, where $(N, A)$ represents the graph; " $N$ " is the set of nodes; " $A$ " is the set of arcs and " $D$ " represents the set of probability distributions that are associated to each node. When a node is not a root node, i.e. when it has some parent nodes, the probability distribution is a conditional probability distribution that quantifies the probabilistic dependency between this node and its parents. A discrete random variable $X$ is represented by a node $X \in N$ with a finite number $(M)$ of mutually exclusive states $S_{X}:\left\{s_{1}^{X}, \ldots s_{M}^{X}\right\}$. The vector $P(X)$ denotes a probability distribution over these states as equation (1):

$$
\begin{array}{r}
P(X)=\left[P\left(X=s_{1}^{X}\right) \ldots P\left(X=s_{m}^{X}\right) \ldots P\left(X=s_{M}^{X}\right)\right] \\
P\left(X=s_{m}^{X}\right) \geq 0
\end{array}
$$

with

$$
\sum_{m=1}^{M} P\left(X=s_{m}^{X}\right)=1
$$

where $P\left(X=s_{m}^{X}\right)$ is the marginal probability of node $X$ being in state $s_{m}^{X}$. In the graph depicted in Figure 1, nodes $X$ and $Y$ are linked by an arc. If $(X, Y) \in A$ and $(Y, X) \notin A$ then $X$ is considered as a parent of $Y$. The set of the parents of node $Y$ is defined as $p a(Y)=X$.

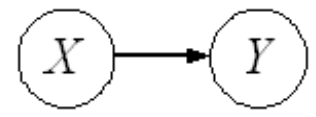

Figure 1. Basic Bayesian Network

In this work, the set $D$ is represented with conditional probability tables. Each node has an associated conditional probability table. For instance, in Figure 1, nodes $X$ and $Y$ are defined over the sets $S_{X}:\left\{s_{1}^{X}, \ldots s_{M}^{X}\right\}$ and $S_{Y}:\left\{s_{1}^{Y}, \ldots s_{L}^{Y}\right\}$. The conditional probability table of $Y$ is then defined by the conditional probabilities $P(Y \mid X)$ over each $Y$ state knowing its parent states $X$. Concerning the root nodes, i.e. those with no parents, the conditional probability table contains only a row describing the $a$ priori probability of each state.

Various inference algorithms can be used to compute the marginal probabilities for each unobserved node given information on the states of the set of observed nodes. The most classical one relies on the use of a junction tree (see [16], pp. 76). Inference in Bayesian Networks [17] allows to update the probabilities of the other variables by taking into account any state variable observation (an event). Without any event observation, the computation is based on a priori probabilities. When observations are given, this knowledge is integrated into the network and all the probabilities are updated accordingly.

Knowledge is formalized as evidence. A hard evidence of the random variable $X$ indicates that the variable state is one of the states $S_{X}:\left\{s_{1}^{X}, \ldots s_{M}^{X}\right\}$. For instance $X$ is in state $s_{1}^{X}: P\left(X=s_{1}^{X}\right)=1$ and $P\left(X=s_{m \neq 1}^{X}\right)=$ 0 . Nevertheless, when this knowledge is uncertain, soft evidence can be used (see [18]). A soft evidence for a node $X$ corresponds to an update of the prior probability values for the states of this node. For example, if $X$ is in state $s_{1}^{X}$ or $s_{M}^{X}$ then the corresponding prior probability are $P\left(X=s_{1}^{X}\right)=0.5, P\left(X=s_{M}^{x}\right)=0.5$ and the others are $P\left(X=s_{m \neq\{1, M\}}^{X}\right)=0$.

\section{A. Bayesian Networks to model reliability}

In order to model the reliability of systems, we propose to use Bayesian Networks in the same way as Fault Trees [19], [20] even if Bayesian Networks are able to do more. We consider components or function with binary states (1) and the same assumptions as fault trees.

A fault tree allows to describe the propagation mechanism of the failure into the system. The system reliability or availability is modeled according to the assumption of independence between the events affecting the entities (hypothesis (a), see chapter 7 in [21]). Whereas a classical model of the parallel structure, as shown in Figure 2, is based on a fault tree, the modeling with a Bayesian Network is realized with a single ' $V$ structure' as depicted in Figure 3. Note that the structure is the same for serial configurations. 


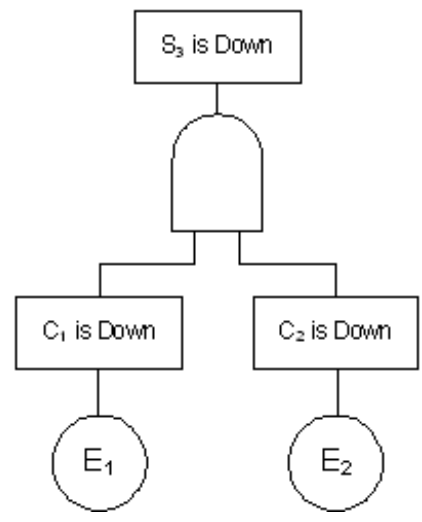

Figure 2. Classical fault tree model of parallel components

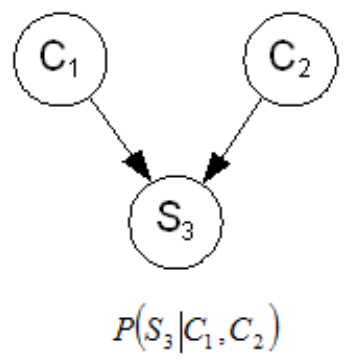

Figure 3. Equivalent Bayesian Network of parallel structure

The conditional probabilities table (CPT) $P\left(S_{3} \mid C_{1}, C_{2}\right)$ contains the conditional probabilities (Table I) which explain the failure propagation mechanism through the functional architecture of the system.

\begin{tabular}{|c|c|c|c|c|c|}
\hline \multirow{2}{*}{} & $C_{1}$ & \multicolumn{2}{|c|}{$U p$} & \multicolumn{2}{c|}{ Down } \\
\cline { 2 - 6 } & $C_{2}$ & $\{U p\}$ & $\{$ Down $\}$ & $\{U p\}$ & $\{$ Down $\}$ \\
\hline \multirow{2}{*}{$S_{3}$} & $\{U p\}$ & 1 & 1 & 1 & 0 \\
\cline { 2 - 6 } & $\{$ Down $\}$ & 0 & 0 & 0 & 1 \\
\hline
\end{tabular}

TABLE I.

Conditional Probabilities TABLE of PARALLEL STRUCTURE

Therefore, the conditional probabilities table is automatically defined by the type of the gate. These conditional probabilities tables are a priori given and probabilities are equal to 0 or 1 since the logic of the failure propagation is deterministic. Then, to compute the reliability of the function $\mathrm{S}_{3}$, as shown on Figure 3, when events on a component are considered statistically independent [8], [22], the following equation is used:

$$
\begin{aligned}
P\left(S_{3}=\{U p\}\right) & =\prod_{i=1}^{2} P\left(C_{i}=\{U p\}\right) \\
& =1-\prod_{i=1}^{2}\left(1-P\left(C_{i}=\{\text { Down }\}\right)\right)
\end{aligned}
$$

\section{B. Bayesian Network to model dependent failure modes and uncertain propagations}

Bayesian Networks provide a powerful mathematical formalism to model complex stochastic processes because this modeling method is based on a Direct Acyclic Graph model and not a tree. Thus, the hypothesis of independence between events (failures) made for a fault tree is not necessary. Indeed, the Bayesian Network allows to compute the exact influence of dependent variables to the system reliability. Moreover, thanks to the conditional probabilities table (CPT), Bayesian Networks provide a model of the propagation of several failure modes in the system.

The variables are not necessary Boolean therefore it is possible to represent a system composed of some entities with several failure modes in a factorized form. Failure Mode Effects Analysis method (FMEA) [8] allows to determine the failure modes associated with a component. Therefore, the states (considered as exhaustive) of a component node are, for instance: $\{U p\}$ the component is available, $\left\{\right.$ Down $\left._{1}\right\}$ : the component is unavailable due to failure $1,\left\{\right.$ Down $\left._{2}\right\}$ : the component is unavailable due to failure 2 , and so on. It is also possible to introduce uncertainty by setting conditional probabilities in the interval of value $[0,1]$.

The states of function $S_{3}$ are defined by failure modes. For instance, node $S_{3}$ in the Bayesian Network (Figure 3) takes the following states: $\{U p\}$ or $\{$ Down $\}$. No prior probability is associated with these states because they are computed according to the states of their parents, i.e. the causes described by $C_{i}$ nodes. The conditional probability table of function $S_{3}$ (Table II) is defined by using the columns of the causes and the failure modes of the FMEA analysis. Nevertheless, a Bayesian Network representation can turn out to be useful insofar as a combination of causes (for instance $C_{1}=\left\{\right.$ Down $\left._{2}\right\}$ and $\left.C_{2}=\{U p\}\right)$ can lead to several failure modes of the function with different probabilities. In Table II, the uncertainty is represented by the probability distribution

\begin{tabular}{|c|c|c|c|c|c|}
\hline & $C_{1}$ & \multicolumn{2}{|c|}{$U p$} & \multicolumn{2}{|c|}{ Down $_{1}$} \\
\hline & $C_{2}$ & $\{U p\}$ & $\{$ Down $\}$ & $\{U p\}$ & $\{$ Down $\}$ \\
\hline \multirow[t]{2}{*}{$S_{3}$} & $\{U p\}$ & 1 & 1 & 1 & 0 \\
\hline & $\{$ Down $\}$ & 0 & 0 & 0 & 1 \\
\hline & $C_{1}$ & \multicolumn{2}{|c|}{ Down $_{2}$} & & \\
\hline & $C_{2}$ & $\{U p\}$ & $\{$ Down $\}$ & & \\
\hline \multirow[t]{2}{*}{$S_{3}$} & $\{U p\}$ & 0.2 & 0 & & \\
\hline & $\{$ Down $\}$ & 0.8 & 1 & & \\
\hline
\end{tabular}
$(0.2 ; 0.8)$.

TABLE II.

UNCERTAIN PROPAGATION IN CPT OF PARALLEL STRUCTURE

As it is known in the FMEA analysis, a failure mode can induce other failure modes according to the mechanism of failure propagation through the system. The Bayesian Network representation is useful to model this kind of propagation but its development has to be rigorously organized [23]. 


\section{Conditional probability tables for AND/OR gates}

As specified previously, the conditional probability tables represent the propagation process of the failures according to the functional architecture of the system. The AND/OR gates are mainly encountered in reliability analyzes. This is why the definition of conditional probability tables that correspond to these two modes of failure propagation is essential in this work. The conditional probability table of an AND gate is provided by Table III and Table IV present an OR gate.

\begin{tabular}{|c|c|c|c|c|c|}
\hline \multirow{2}{*}{} & $C_{1}$ & \multicolumn{2}{|c|}{$U p$} & \multicolumn{2}{c|}{ Down } \\
\cline { 2 - 6 } & $C_{2}$ & $\{U p\}$ & $\{$ Down $\}$ & $\{U p\}$ & $\{$ Down $\}$ \\
\hline \multirow{2}{*}{$S_{3}$} & $\{U p\}$ & 1 & 0 & 0 & 0 \\
\cline { 2 - 6 } & $\{$ Down $\}$ & 0 & 1 & 1 & 1 \\
\hline
\end{tabular}

TABLE III.

CONDITIONAL PROBABILITY TABLE FOR A AND GATE

\begin{tabular}{|c|c|c|c|c|c|}
\hline \multirow{2}{*}{} & $C_{1}$ & \multicolumn{2}{|c|}{$U p$} & \multicolumn{2}{c|}{ Down } \\
\cline { 2 - 6 } & $C_{1}$ & $\{U p\}$ & $\{$ Down $\}$ & $\{U p\}$ & $\{$ Down $\}$ \\
\hline \multirow{2}{*}{$S_{3}$} & $\{U p\}$ & 1 & 1 & 1 & 0 \\
\cline { 2 - 6 } & $\{$ Down $\}$ & 0 & 0 & 0 & 1 \\
\hline
\end{tabular}

TABLE IV.

CONDITIONAL PROBABILITY TABLE FOR AN OR GATE

\section{Conditional probability table for $k / n$ gates}

The $k / n$ gate is a particular useful gate to model the reliability of some parts of a system. The principle is that $k$ elements among $n$ should be on state $\{U p\}$ to warrant the subsystem to be in state $\{U p\}$. This special gate is easily modeled in Bayesian Networks with the appropriate conditional probability table. For instance, Table $\mathrm{V}$ is the conditional probability table for a $2 / 3$ gate.

\begin{tabular}{|c|c|c|c|c|c|}
\hline & $C_{1}$ & \multicolumn{4}{|c|}{$U p$} \\
\hline & $C_{2}$ & \multicolumn{2}{|c|}{$U p$} & \multicolumn{2}{|c|}{ Down } \\
\hline & $C_{3}$ & $\{U p\}$ & $\{$ Down $\}$ & $\{U p\}$ & $\{$ Down $\}$ \\
\hline \multirow[t]{2}{*}{$S_{3}$} & $\{U p\}$ & 1 & 1 & 1 & 0 \\
\hline & $\{$ Down $\}$ & 0 & 0 & 0 & 1 \\
\hline & $C_{1}$ & \multicolumn{4}{|c|}{$\overline{\{\text { Down }\}}$} \\
\hline & $C_{2}$ & \multicolumn{2}{|c|}{$U p$} & \multicolumn{2}{|c|}{ Down } \\
\hline & $C_{3}$ & $\{U p\}$ & $\{$ Down $\}$ & $\{U p\}$ & $\{$ Down $\}$ \\
\hline \multirow[t]{2}{*}{$S_{3}$} & $\{U p\}$ & 1 & 0 & 0 & 0 \\
\hline & $\{$ Down $\}$ & 0 & 1 & 1 & 1 \\
\hline
\end{tabular}

TABLE V.

CONDITIONAL PROBABILITY TABLE FOR 2/3 GATE

As shown with Tables II, III and IV, the Bayesian Networks make it easy to explain the propagation mechanisms of the system failures. However, the states of the components are necessarily disjoint and known $(\{U p\}$ and $\{$ Down $\}$ for the binary case in our previous examples). But, in operation, the components do not respect perfectly the theoretical laws of degradation. Moreover, all the components are not simultaneously inspected by the maintenance team. In these situations, we are faced to missing data or inconsistencies between the Bayesian Network reliability model of the system and the reality. The probabilistic framework on which are based Bayesian Networks is not suitable to take into account these two problems. Indeed, the first case leads to a censure or a completion with a soft evidence using an uniform distribution as defined in the section II. The second case leads to the censure of the data.

The Evidence theory is, on some points, close to the probability theory. It offers a more open formal framework to deal with the problems quoted previously. However, there is a lack of industrial tools allowing its implementation, only some toolboxes exist [24], [25]. Our goal is thus to use the flexibility offered by the Evidence theory for the incompleteness and inconsistency problems and to combine it with the modeling power of Bayesian Networks for the reliability studies.

\section{EVIDENCE THEORY FORMALISM}

The evidence theory, sometimes called belief functions theory, has been initiated by Dempster with its works on milestones superior and inferior bounds of a family of probability distributions [26] then reinforced by Shafer [27]. Different processing models of imperfect information have thus appeared [25]:

- The probability based belief function theory

- Upper and lower model [28]

- Dempster-Shafer Theory

- Hint Model of Kholas and Monney [29].

- The probabilistic argumentation system of Haenni, Kholas and Lehman [30]

- The non-probabilistic belief function theory

- The theory presented in Shafer's book [27]

- Transferable Belief Model [14], [31]

On some aspect, the evidence theory can be seen close to the probability theory. Moreover, the Generalized Bayesian Theorem can be found in the evidence theory [25]. This theorem presents a major interest for the applications of the Dempster-Shafer theory by Bayesian Networks algorithms.

Within this formal framework, there is an allocation process of a quantity (belief mass) ranging between 0 and 1 to the different proposals (basic belief assignment). This allocation process is rather close to that of the probabilistic framework, but is different by the possibility to allocate a quantity to all tuples of proposals.

\section{A. Basic belief assignment}

The allocation process in the probability framework follows the equation below:

$$
\begin{array}{r}
P: 2^{\Omega} \rightarrow[0,1] \text { such that: } \\
P(\emptyset)=0 \\
\sum_{s_{i}^{X} \in \Omega} P\left(X=s_{i}^{X}\right)=1
\end{array}
$$


where the frame of discernment $\Omega$ is composed of each disjoint proposals $s_{i}^{X}$ (states in reliability studies) and $\emptyset$. This frame of discernment serves to elaborate prior probabilities on component states and posterior probabilities on subsystem states.

In the evidence theory, the basic belief assignment follows the function below:

$$
\begin{array}{r}
M: 2^{\Omega} \rightarrow[0,1] \text { such that: } \\
M(\emptyset)=0 \\
\sum_{s_{i}^{X} \in \Omega} M\left(X=s_{i}^{X}\right)=1
\end{array}
$$

where the frame of discernment $\Omega$ is composed of all possible tuples of disjoint proposals $s_{i}^{X}$. This frame of discernment serves to elaborate prior masses on component states and posterior masses on subsystem states.

As mentioned previously, the reliability studies made in this paper concerns binary state components. The corresponding frame of discernment is described by the following equations:

$$
\Omega=\{U p, \text { Down }\}
$$

And $2^{\Omega}=\{\emptyset ;\{U p\} ;\{$ Down $\},\{U p$, Down $\}\}$, under the constraints (6) and (7).

Thus, the possibility to allocate a quantity to the proposal $\{U p$, Down $\}$ softens the probability framework.

The role of this reasoning modality is to characterize our ignorance on the real state of the component without commitment. It means that the component can be in the state $\{U p\}$ or $\{D o w n\}$. Of course, it should not be considered that the component can be in the two states simultaneously. This ignorance characterizes the epistemic uncertainty and $\{U p, D o w n\}$ the epistemic state.

As mentioned before, hypotheses (6) and (7) are verified in our study and in the general case of reliability study. When these assumptions are not verified the framework concerns the open world concept which is out of the scope of this paper [14], [30]. This concept can be very interesting in more complex studies.

Note that we consider binary state components in previous equations. These assumptions are those generally used in quantitative reliability analysis. Currently, reliability engineers extend their works to multistate components. In this case, the number of combinations in the allocation function $M$ increases and consequently the number of epistemic modalities increases too. This is a well-known practice problem in evidence theory. Nevertheless, in the case of reliability studies based on databases, basic belief assignments are computed directly from data and this computing mitigates engineers' efforts.

\section{B. Plausibility and belief functions}

In the evidence theory, two main functions help us to obtain information about the uncertainty in the knowledge, the plausibility and belief functions.
Plausibility function characterizes the degree in which a proposal $X=s_{i}^{X}$ is plausible based on available evidence $X=s_{j}^{X}$ expressed by each basic belief mass that contributes to the realization of $X=s_{i}^{X}$. This function is defined as pls : $2^{\Omega} \rightarrow[0,1]$ by the following equation:

$$
p l s\left(X=s_{i}^{X}\right)=\sum_{s_{j}^{X} \mid s_{i}^{X} \cap s_{j}^{X} \neq \emptyset} M\left(X=s_{j}^{X}\right)
$$

The dual function is the belief function that characterizes the degree in which a proposal $X=s_{i}^{X}$ is believable based on available evidence expressed by each basic belief mass assigned on the frame of discernment. This function is defined as bel : $2^{\Omega} \rightarrow[0,1]$ by the following equation:

$$
\operatorname{bel}\left(X=s_{i}^{X}\right)=\sum_{s_{j}^{X} \mid s_{j}^{X} \subseteq s_{i}^{X}} M\left(X=s_{j}^{X}\right)
$$

\section{Probability boxing}

By using equations (8) and (9), we obtain an interesting characteristic which is the boxing of the probability by the belief and plausibility functions [27], [32].

$$
\operatorname{bel}\left(X=s_{i}^{X}\right) \leq P\left(X=s_{i}^{X}\right) \leq p l s\left(X=s_{i}^{X}\right)
$$

The property (10) is well known, Shafer has already defined it in his works in 1976 [27]. Many authors have used this direct relation between interval [bel,pls] and basic belief assignment [33]-[35]. Some authors [12], [34], [36] prefer taking the problem by defining interval valued probability rather than assigning belief masses.

Two reasons make us reject this last approach. On the one hand, the uncertainty on the state of a component is not expressed in an epistemic manner with interval valued probabilities. It concerns an imprecision on the value of the failure probability and not an expression of our ignorance about the allocation of masses for some events. It concerns a calculation of imprecise probabilities [12], [37], a random uncertainty problem rather than a calculation with the epistemic uncertainty. On the other hand, Bayesian Networks do not allow this mode of processing.

\section{Decision, pignistic or plausibility transformation}

In his works, Smets [25] argue that evidence theory is a two level mental model in order to distinguish between two aspects of belief, belief as weighted opinions and belief for decision making. The two levels are :

- the credal level where beliefs are entertained,

- the pignistic level where beliefs are used to make decision

Beliefs at the credal level are represented by belief function or basic belief assignments. When decision must be made, the belief held at the credal level induced a pignistic probability at the pignistic level through a pignistic transformation [38], [39]. Shenoy [40] has criticized 
the pignistic transformation because it can induces inconsistencies in some particular cases. He proposed a more convenient transformation, the plausibility transformation.

\section{E. Discussion}

As mentioned previously, the evidence theory formalism is close to the probability one, but it takes into account inconsistencies and incompleteness in a better way. The frame of discernment allows to define how many events concern the state $\{U p\}$ or the state $\{$ Down $\}$ and how many events can not be affected to the previous cases. In addition, plausibility and belief functions help to compute measures that bound the real value of the probability of failure that is often preferred by reliability engineer in current reliability analysis. The precise value can be obtained by a transformation of belief from the credal level to the pignistic one.

By introducing the allocation process of the evidence theory in Bayesian Networks, new properties are offered to manage incompleteness, inconsistencies and therefore uncertainty in reliability studies. By staying in the credal level, we attempt to keep the epistemic and the aleatory uncertainty in the same model. We are able to draw them, to evaluate their impact on the system reliability. Moreover, the transformation from the credal level to the pignistic one truncates our knowledge about the system reliability and does not help the maintenance engineer to manage his actions according the uncertainty and the risks during the system exploitation.

The goal is to produce an Evidential Network by adapting the propagation mechanism of Bayesian Networks according to the main concepts of the evidence theory previously defined.

\section{EVIDENTIAL Networks TO MODEL RELIABiLity}

In order to use Bayesian Networks tools with the evidence theory, we should adapt the conditional probability tables and prior probabilities.

\section{A. Conditional mass tables}

This adaptation consists in the transformation of the conditional probability tables into conditional mass table in order to integrate the basic belief assignment and to describe the propagation mechanism of failures.

On the base of Tables III, IV and V the conditional mass tables for AND, OR and $k / n$ gates should be designed. The conditional probability for $\{U p\}$ and $\{$ Down $\}$ modalities can be used directly and efforts should be made on the $\{U p$, Down $\}$ modality.

As mentioned previously, the modality $\{U p, D o w n\}$ characterizes our ignorance about the component state. As the component can take the two defined states only, the conditional masses of $\{U p, D o w n\}$ in the conditional mass tables are defined by the Boolean logic.

For instance, let's consider an AND gate. If component 1 is in state $\{U p\}$ and component 2 is in state $\{U p\}$ then output $S_{3}$ is in state $\{U p\}$, but if component 2 is in state
$\{$ Down $\}$ the output $S_{3}$ is in state $\{$ Down $\}$. Now let's imagine that the state of component 2 is in the epistemic state and thus can be $\{U p\}$ or $\{D o w n\}$, the output $S_{3}$ can also be in the two states $\{U p\}$ or $\{$ Down $\}$. We express this ignorance on the state of $S_{3}$ by affecting a conditional mass on the epistemic state $\{U p$, Down $\}$.

By continuing this reasoning, the conditional mass table for an AND gate is established and described in Table VI.

\begin{tabular}{|c|c|c|c|c|}
\hline & & \multicolumn{3}{|c|}{$S_{3}$} \\
\hline$C_{1}$ & $C_{2}$ & $\{U p\}$ & $\{$ Down $\}$ & $\{U p$, Down $\}$ \\
\hline \multirow{3}{*}{$U p$} & $\{U p\}$ & 1 & 0 & 0 \\
\hline & $\{$ Down $\}$ & 0 & 1 & 0 \\
\hline & $\{U p$, Down $\}$ & 0 & 0 & 1 \\
\hline \multirow{3}{*}{ Down } & $\{U p\}$ & 0 & 1 & 0 \\
\hline & $\{$ Down $\}$ & 0 & 1 & 0 \\
\hline & $\{U p$, Down $\}$ & 0 & 1 & 0 \\
\hline \multirow{3}{*}{$U p$, Down } & $\{U p\}$ & 0 & 0 & 1 \\
\hline & $\{$ Down $\}$ & 0 & 1 & 0 \\
\hline & $\{U p$, Down $\}$ & 0 & 0 & 1 \\
\hline
\end{tabular}

TABLE VI.

Conditional Mass TABle For An AND gate

The same reasoning is carried out for the construction of the conditional mass table of an OR gate given in Table VII.

\begin{tabular}{|c|c|c|c|c|}
\hline & & \multicolumn{4}{|c|}{$S_{3}$} \\
\hline$C_{1}$ & $C_{2}$ & $\{U p\}$ & $\{$ Down $\}$ & $\{$ Up, Down $\}$ \\
\hline \multirow{3}{*}{$U p$} & $\{$ Up $\}$ & 1 & 0 & 0 \\
\cline { 2 - 5 } & $\{$ Down $\}$ & 1 & 0 & 0 \\
\cline { 2 - 5 } & $\{U p$, Down $\}$ & 1 & 0 & 0 \\
\hline \multirow{3}{*}{ Down } & $\{$ U $\}$ & 1 & 0 & 0 \\
\cline { 2 - 5 } & $\{$ Down $\}$ & 0 & 1 & 0 \\
\cline { 2 - 5 } & $\{U p$, Down $\}$ & 0 & 0 & 1 \\
\hline \multirow{3}{*}{ Up, Down } & $\{$ Up $\}$ & 1 & 0 & 0 \\
\cline { 2 - 5 } & $\{$ Down $\}$ & 0 & 0 & 1 \\
\cline { 2 - 5 } & $\{U p$, Down $\}$ & 0 & 0 & 1 \\
\hline
\end{tabular}

TABLE VII.

Conditional Mass TABle for an OR Gate

The development of these conditional mass tables makes possible to model the propagation of the failure within the model of the system reliability but also the way in which uncertainty is propagated on the knowledge of the state of the components. These propagations will take place from parent nodes or root nodes to child nodes through the network in order to characterize the reliability of the complete system.

\section{B. Conditional Mass Table for $k / n$ gate}

Like the case of AND/OR gates, the conditional probability table of the $k / n$ gate should be translated to integrate the Evidential Network with the correct conditional mass table. The specific $\{U p$, Down $\}$ modality is introduced for each component state and also the output.

For instance, let's consider a $2 / 3$ gate. When two components from the three are in the same state, the output is in the state of the majority of components. In addition, if 2 components among the three are in opposite states $\{U p\}$ and $\{D o w n\}$, the state of the third 
component implies the state of the output. Thus, if the state of this last component is uncertain, the output is also uncertain. The following table gives the conditional mass table. Please, note that for convenience we use $\{U, D\}$ in place of $\{U p, D o w n\}$ in Tables VIII to IX.

\begin{tabular}{|c|c|c|c|c|c|}
\hline & & & \multicolumn{3}{|c|}{$S_{3}$} \\
\hline$C_{1}$ & $C_{2}$ & $C_{3}$ & $\{U p\}$ & $\{$ Down $\}$ & $\{U, D\}$ \\
\hline \multirow{9}{*}{$U p$} & \multirow{3}{*}{$U p$} & $\{U p\}$ & 1 & 0 & 0 \\
\hline & & $\{$ Down $\}$ & 1 & 0 & 0 \\
\hline & & $\{U, D\}$ & 1 & 0 & 0 \\
\hline & \multirow{3}{*}{ Down } & $\{U p\}$ & 1 & 0 & 0 \\
\hline & & $\{$ Down $\}$ & 0 & 1 & 0 \\
\hline & & $\{U, D\}$ & 0 & 0 & 1 \\
\hline & \multirow{3}{*}{$U, D$} & $\{U p\}$ & 1 & 0 & 0 \\
\hline & & $\{$ Down $\}$ & 0 & 0 & 1 \\
\hline & & $\{U, D\}$ & 0 & 0 & 1 \\
\hline \multirow{9}{*}{ Down } & \multirow{3}{*}{$U p$} & $\{U p\}$ & 1 & 0 & 0 \\
\hline & & $\{$ Down $\}$ & 0 & 1 & 0 \\
\hline & & $\{U, D\}$ & 0 & 0 & 1 \\
\hline & \multirow{3}{*}{ Down } & $\{U p\}$ & 0 & 1 & 0 \\
\hline & & $\{$ Down $\}$ & 0 & 1 & 0 \\
\hline & & $\{U, D\}$ & 0 & 1 & 0 \\
\hline & \multirow{3}{*}{$U, D$} & $\{U p\}$ & 0 & 0 & 1 \\
\hline & & $\{$ Down $\}$ & 0 & 1 & 0 \\
\hline & & $\{U, D\}$ & 0 & 0 & 1 \\
\hline \multirow{9}{*}{$U, D$} & \multirow{3}{*}{$U p$} & $\{U p\}$ & 1 & 0 & 0 \\
\hline & & $\{$ Down $\}$ & 0 & 0 & 1 \\
\hline & & $\{U, D\}$ & 0 & 0 & 1 \\
\hline & \multirow{3}{*}{ Down } & $\{U p\}$ & 0 & 0 & 1 \\
\hline & & $\{$ Down $\}$ & 0 & 1 & 0 \\
\hline & & $\{U, D\}$ & 0 & 0 & 1 \\
\hline & \multirow{3}{*}{$U, D$} & $\{U p\}$ & 0 & 0 & 1 \\
\hline & & $\{$ Down $\}$ & 0 & 0 & 1 \\
\hline & & $\{U, D\}$ & 0 & 0 & 1 \\
\hline
\end{tabular}

TABLE VIII.

CONDITIONAL PROBABILITY TABLE FOR 2/3 GATE

\section{Plausibility and belief functions}

To implement the computation of belief and plausibility functions within the simulation tool of Bayesian Networks, we should separate their computing in two different nodes (bel,pls). This solution allows to release the additive constraint (see eq. 7) inherent to Bayesian Networks algorithm which does not exist in the general framework of the evidence theory. The conditional mass table (Table IX) converts relation 9 into the Bayesian Network to compute the plausibility that the system is in state $\{U p\}$. In addition, note that we have introduce the modality NotBelieve to allow the Bayesian Network node to respect the additive constraint.

\begin{tabular}{|c|c|c|c|c|}
\hline & System state & $\{U p\}$ & $\{$ Down $\}$ & $\{U, D\}$ \\
\hline bel $\begin{array}{c}\text { System state } \\
=\{U p\})\end{array}$ & Believe & 1 & 0 & 0 \\
\cline { 2 - 5 } & NotBelieve & 0 & 1 & 1 \\
\hline
\end{tabular}

TABLE IX

TABLE OF bel(System state $=\{U p\})$

On the same way, we define the conditional mass table (Table X) to compute the plausibility function of the system (eq. 8) and introduce the modality Notplausibility to allow the Bayesian Network node to respect the additive constraint.

\begin{tabular}{|c|c|c|c|c|}
\hline & System state & $\{U p\}$ & $\{$ Down $\}$ & $\{U, D\}$ \\
\hline \multirow{2}{*}{\begin{tabular}{c} 
pls $\begin{array}{c}\text { System state } \\
=\{U p\})\end{array}$ \\
\cline { 2 - 5 }
\end{tabular}} & Plausibility & 1 & 0 & 0 \\
\cline { 2 - 5 } & Not plausibility & 0 & 1 & 1 \\
\hline
\end{tabular}

TABLE X.

TABLE OF pls (system state $=\{U p\})$

Besides, we can note that these tables can be used to compute plausibility and belief functions on each component or node of the network in order to give information on probability boxes that bound the real value of the probability for subsystems.

\section{Applications}

We dedicate this section to examples in order to show how the Evidential Network computes the reliability of complex systems under uncertainty. Our first example is the simulation of a simple system with a $2 / 3$ gate to evaluate the propagation mechanism. The second example is the bridge system previously defined by Torres-Toledano in [10]. The third example is a complex system defined by Kamat and Riley [41] for the evaluation of quantitative reliability method with Monte Carlo simulations. For each example, the Evidential Network that model the system reliability model is presented and the according basic belief assignments (bar graphes) are shown on the right side.

\section{A. system with $2 / 3$ gate}

For this first example, we propose to simulate a $k / n$ gate. The goal is to show the ability of the Evidential Network to compute the system reliability. The Bayesian Network of a $2 / 3$ gate is translated into the Bayesian Network tool Bayesialab(c) with the structure presented on Figure 4.
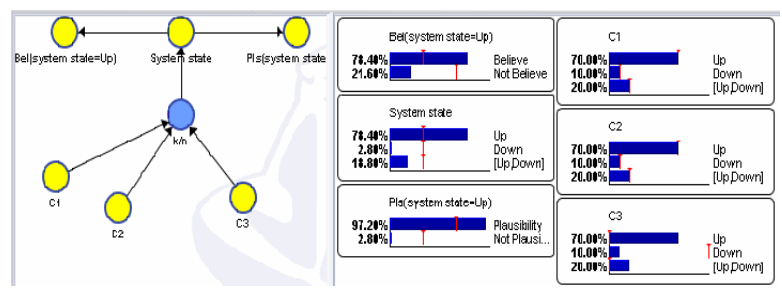

Figure 4. k/n gate in Bayesialab.

We consider each component with the same basic belief assignment arbitrarily chosen. The elementary events that drive the components from state $\{U p\}$ to state $\{$ Down $\}$ are independent. The system is homogeneous and no repair is considered. The system reliability is given theoretically by the following equation:

$$
R\left(T_{F}\right)=\sum_{k=m}^{n} C_{n}^{k} r^{k}(1-r)^{n-k} \text { with } C_{n}^{k}=\frac{n !}{k !(n-k) !}
$$


If we consider the probability value of the state $\{U p\}$ is $r=0.7$ for each component in order to compute the system reliability then by using the equation 11 , we found $R\left(T_{F}\right)=0.784$ where $T_{F}$ is the time of mission.

Note that this value is computed by the Network for the reliability of the system (Figure 4 ). The boxing property is maintained with a $k / n$ gate. Moreover, we can notice that the epistemic uncertainty on each component has a large contribution on the system state.

\section{B. Bridge system}

This system has been previously defined by TorresToledano in [10]. It seems to be quite simple but presents a difficulty in computing the system reliability. The bridge system consists of five components $C_{1}$ to $C_{5}$ (Figure 5). The component failures are independent. The system is homogeneous and no repair is considered.

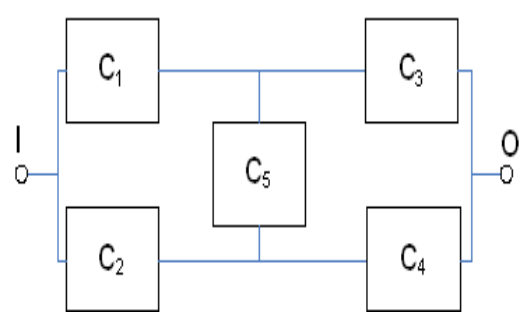

Figure 5. Reliability diagram of the bridge system.

The corresponding Evidential Network is presented on Figure 6 . The network propagates basic belief assignments according to conditional mass tables defined by Table VI and VII.

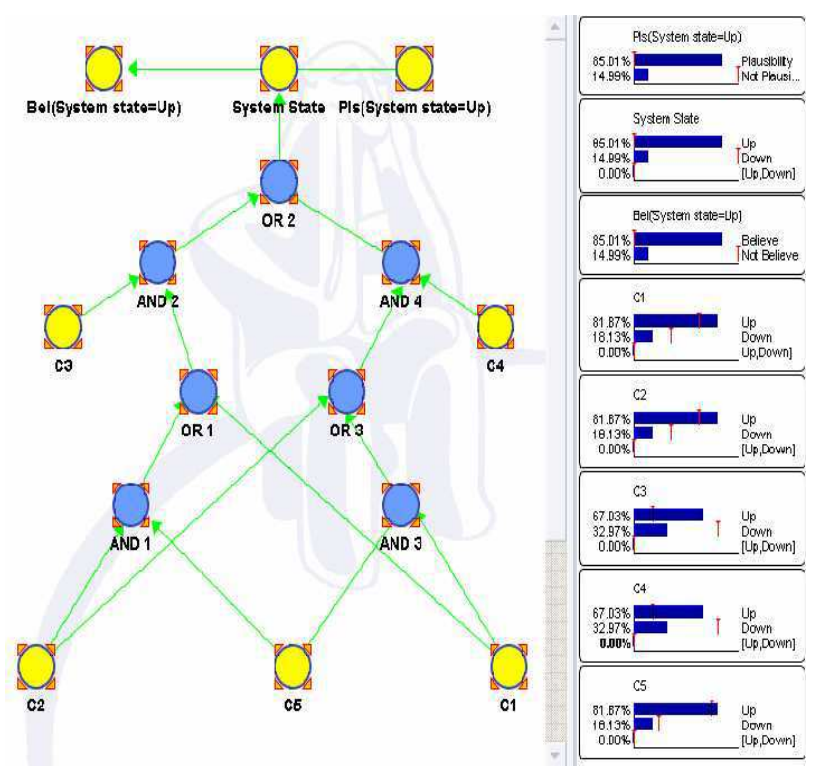

Figure 6. Bridge system reliability model with the Evidential Network.
To evaluate the exactness of the Evidential Network, let us define numerical values for failure rates of each component $\left(\lambda_{1}=\lambda_{2}=\lambda_{5}=10^{-3} h^{-1} ; \lambda_{3}=\lambda_{4}=\right.$ $\left.2.10^{-3} h^{-1}\right)$. We consider the time of mission up to $T_{F}=$ $200 h$, then $R_{1}\left(T_{F}\right)=R_{2}\left(T_{F}\right)=R_{5}\left(T_{F}\right)=0.81873$ and $R_{3}\left(T_{F}\right)=R_{4}\left(T_{F}\right)=0.7032$.

By applying the total probabilities theorem, we obtain the exact value of the system reliability $R_{S}\left(T_{F}\right)=0.85$. If the reliability of the system components is introduce as prior masses in the corresponding nodes of the Evidential Network, the system reliability is given by bel(system state $=\{U p\})$ or pls(system state $=$ $\{U p\})$ as it can be seen on the right part of Figure 6 .

Now, let's consider the Evidence Network with epistemic uncertainty introduced on the state of the component $C_{1}$. The propagation of this uncertainty can be observed in the Bayesian Network. According to the importance of the component in the system and the quantity of uncertainty attached, the epistemic uncertainty on the system reliability can vary importantly. For example, if the mass of epistemic uncertainty $M\left(C_{1}=\{U p\right.$, Down $\left.\}\right)=$ 0.1 is introduced in the prior mass table of $C_{1}$ then:

$$
\begin{aligned}
\text { bel }(\text { System state }=\{U p\}) & =0.84 \\
P(\text { System state }=\{U p\}) & =0.85 \\
\text { pls }(\text { System state }=\{U p\}) & =0.86
\end{aligned}
$$

The basic belief assignment of each component can be observed on Figure7.

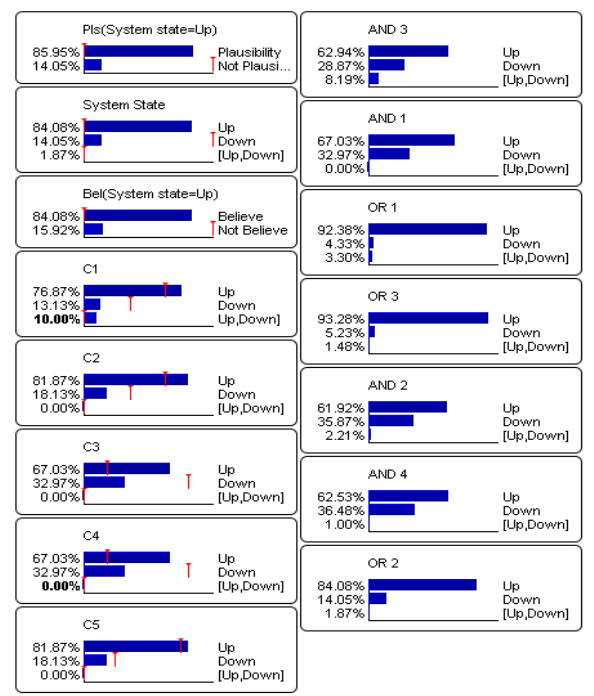

Figure 7. Basic belief assignments with $M\left(C_{1}=\{U p\right.$, Down $\left.\}\right)=$ 0.1

That leads to $M$ (System state $=\{U p$, Down $\})=$ 0.0187. If the uncertainty on $C_{1}$ grows up to 0.3 $M($ System state $=\{U p$, Down $\})$ goes to 0.0561 .

$$
\begin{aligned}
\text { bel }(\text { System state }=\{U p\}) & =0.82 \\
P(\text { System state }=\{U p\}) & =0.85 \\
\text { pls }(\text { System state }=\{U p\}) & =0.88
\end{aligned}
$$


The new basic belief assignment of each component can be observed on Figure 8 .

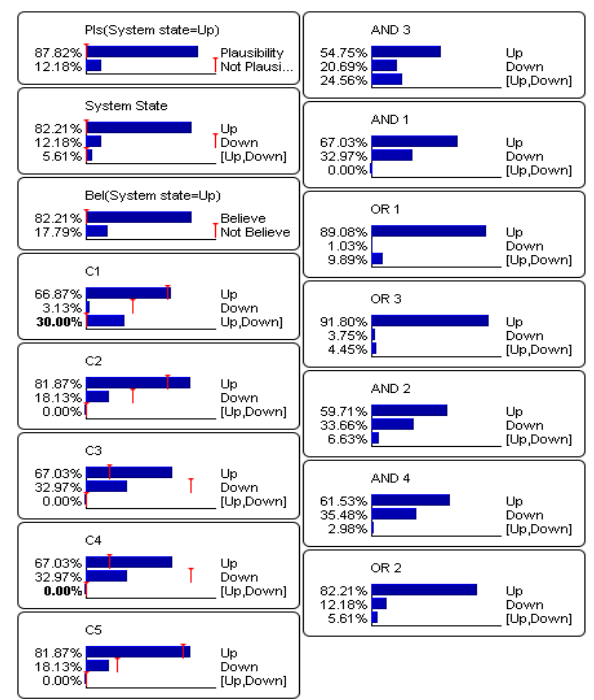

Figure 8. Basic belief assignments with $M\left(C_{1}=\{U p\right.$, Down $\left.\}\right)=$ 0.3

If the basic belief assignment on the system state expresses an epistemic uncertainty then bel and $p l s$ measures are not equal and bound the system reliability. So, further decision can be made according to this uncertainty.

\section{Kamat-Riley system}

Let's consider a more complex system extracted from a study of Kamat and Riley [41] shown on Figure 9. This system is considered as a complex system since it can not be rewritten by a combination of series-parallel subsystems [8]. In order to simplify this example, we consider that all components have the same basic belief assignment for the current mission time. We choose the following value for the reliability of each component: $R_{i}\left(T_{F}\right)=0.81873$

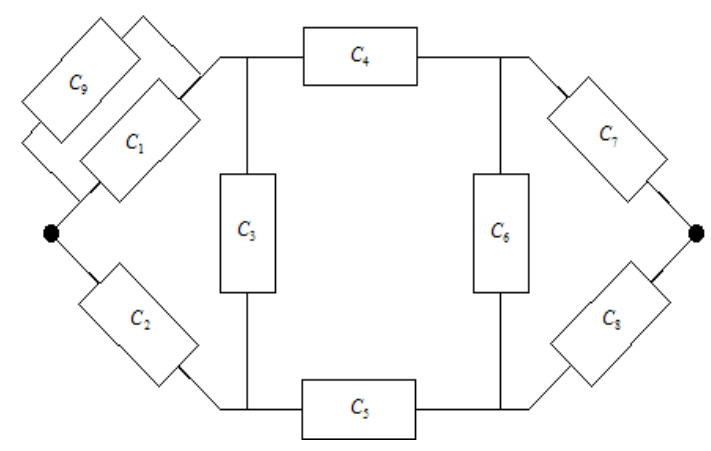

Figure 9. Complex system from a study of Kamat and Riley

By determining all success path of this system, we construct the following Bayesian Network which is the solution to compute the system reliability.

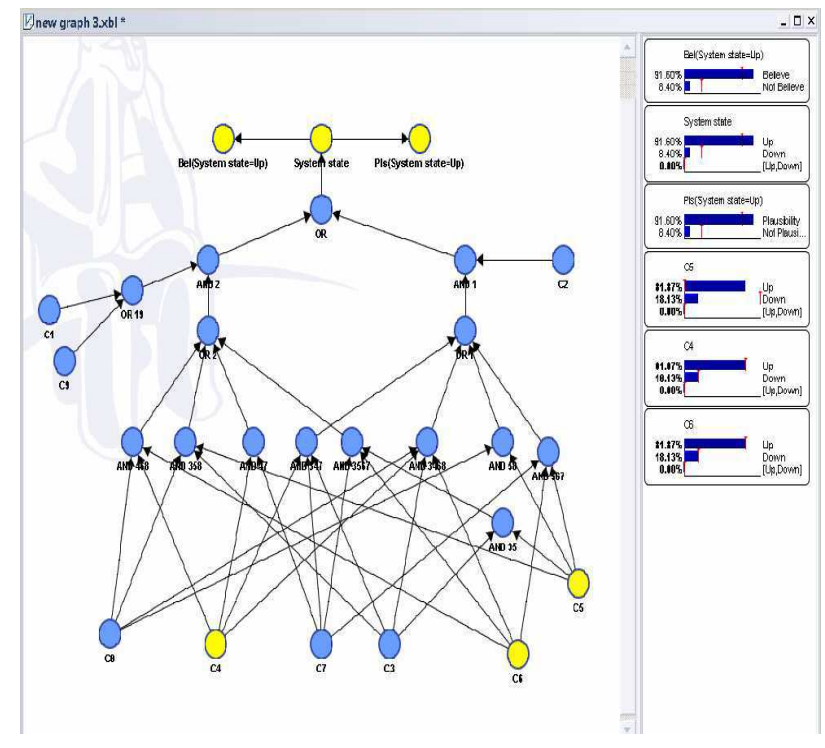

Figure 10. Bayesian Network solution of Kamat-Riley system

As we can see, with 9 components, the system becomes a little bit more complicate. Note that AND/OR gates with multiple inputs are introduced to reduce the number of nodes. Like in the previous example and as waited, we found the boxing property of the probability that the system is $\{U p\}$. Of course, as we don't introduce any epistemic uncertainty on basic belief assignments of the components, the belief and plausibility measures are equal to the probability measure.

Let us introduce an epistemic uncertainty value of 0.20 into the basic belief assignments of $C_{2}, C_{5}$ and $C_{9}$. The resulting basic belief assignment and belief measures of the system reliability can be observed on Figure 11 . This epistemic uncertainty propagates through the network and induces an epistemic uncertainty on the system state. We can notice that even if three components have a large value of epistemic uncertainty, the value for the system remains low. This value depends on the importance of the components in the system and is mitigated by the basic belief assignment of the other components.

Let's consider a new set of basic belief assignments where $M\left(C_{2}=\{U p\}\right) \quad=$ 0.4397, $M\left(C_{2}=\{\right.$ Down $\left.\}\right)=0.2010$ and $M\left(C_{2}=\{U p, D o w n\}\right)=0.3593$. By computing the belief and plausibility measures on the unreliability of $C_{2}$, we obtain bel $\left(C_{2}=\{\right.$ Down $\left.\}\right)=0.2010$ and pls $\left(C_{2}=\{\right.$ Down $\left.\}\right)=0.5603$. We can conclude that the component is plausibly $\{D o w n\}$. In this situation, the maintenance manager can launch an inspection to definitively reduce the uncertainty on this component. The simulation tool allows us to define the state of $C_{2}$ as up for instance and computes the new set of basic belief assignments given this new information as shown on Figure 12. This ability of the simulation tool can be 


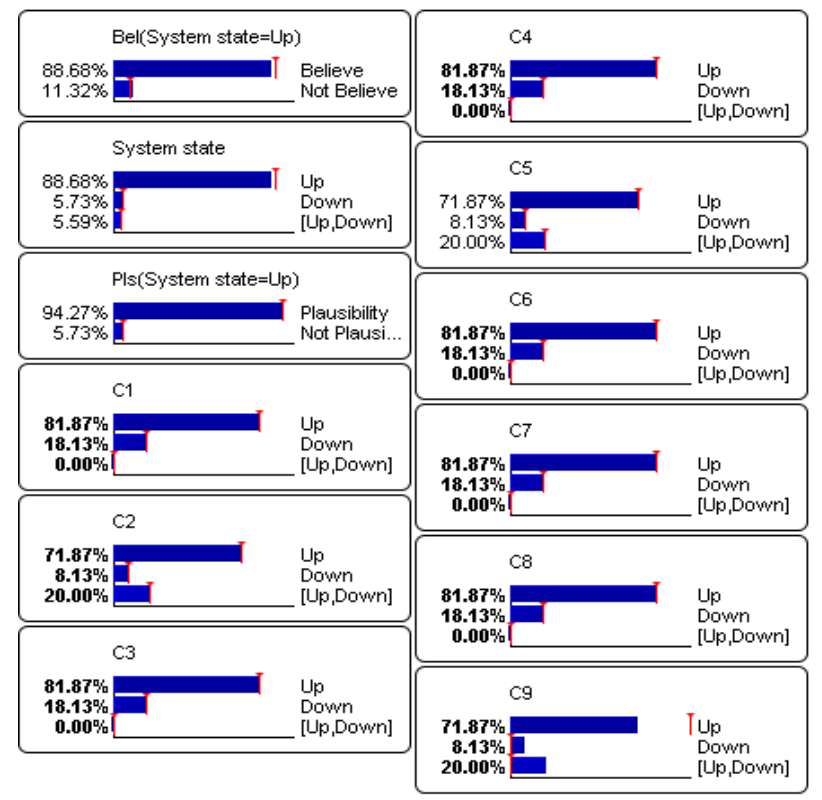

Figure 11. Kamat-Riley system with epistemic uncertainty value set to 0.2 on $C_{2}, C_{5}$ and $C_{9}$.

of a great interest for the maintenance team.

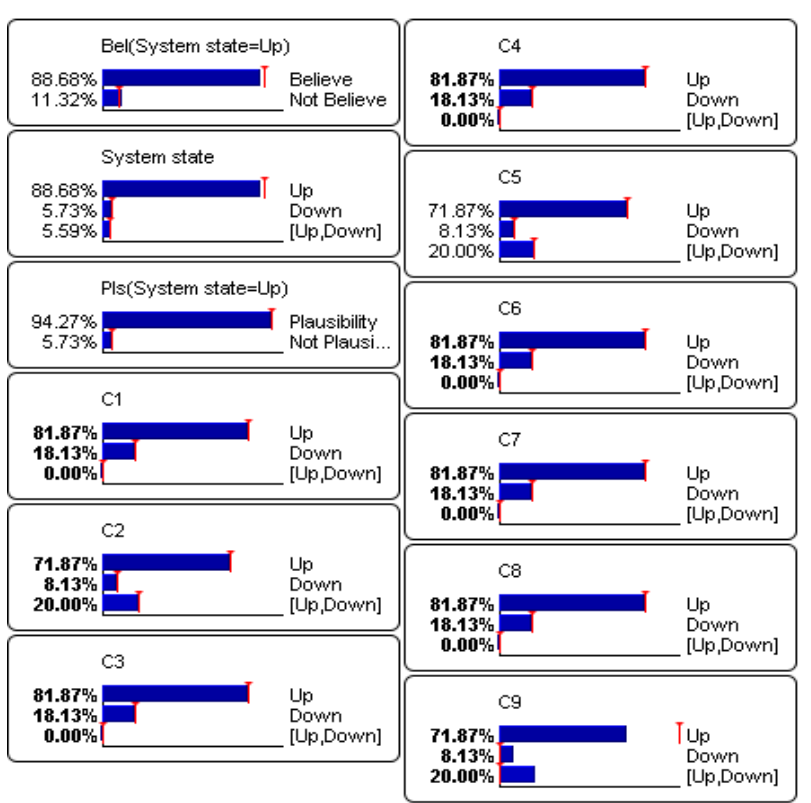

Figure 12. Basic belief assigments with $M\left(C_{2}=\{U p\}\right)=1$

\section{CONCLUSION}

In this paper, the powerful representation and the exactness of Bayesian Networks in reliability studies is shown. In some context like incomplete data in databases or incoherency between data and reliability models, we can use uncensored data with the Dempster Shafer Theory to consider the epistemic uncertainty. The paper shows how basic concepts of the Dempster Shafer Theory can be implemented in Bayesian Networks tools to treat this kind of uncertainty and to extract the most of information from the available data. Moreover, the Dempster-Shafer Theory offers a generalized Bayesian framework which allows us to use Evidential Networks in their original form without modification. It provides a more powerful tool to manage uncertainty in artificial intelligence and approximate reasoning. In the framework of the evidence theory, we recognize a two-level mental model: the credal level where beliefs are held and represented by belief functions and the pignistic level where decisions are made [25]. Many transformation functions can be found: the pignistic transformation defined by Smets [38] or the plausibility transformation [40]. In this paper, we have chosen to work on the credal level only and to manipulate the basic belief assignments and their propagation through Bayesian Networks inference algorithm. The goal is to help the reliability engineer to handle the epistemic uncertainty of the system state according to the epistemic uncertainty of the state of the components. The belief measure informs the reliability engineer about the worst value of the reliability of the system. By running diagnostic or intercausal modes of Bayesian Networks inference algorithm, the reliability engineers can identify the components which introduce the epistemic uncertainty on the state of the system. Then, he can plan all needed inspection to reduce this uncertainty. This ability of Evidence Networks to manage the epistemic uncertainty allows us to investigate problems with time dependence like maintenance policies and we have to work on implementing some transformations to elaborate pignistic decision. Furthermore, the investigation of multistate systems and diagnostic problems with soft evidence can be of great interest for the reliability engineer.

\section{REFERENCES}

[1] S. Yang, N. Hsu, P. Louie, and W. Yeh, "Water distribution network reliability: Connectivity analysis," ASCE Journal of Infrastructure Systems, vol. 2, pp. 62-74, 1996.

[2] — "Water distribution network reliability: Stochastic simulation," ASCE Journal of Infrastructure Systems, vol. 2, pp. 75-83, 1996.

[3] K. Aggarwal, K. Misra, and J. Gupta, "A fast algorithm for reliability evaluation," IEEE Transportation on Reliability, vol. 24, pp. 83-85, 1975.

[4] Y. Asakura and M. Kashiwadani, "Road network reliability caused by daily fluctuation of traffic flow," in Proceedings of the 19th PTRC Summer Annual Meeting, 1991, pp. 7394.

[5] IEC, IEC61508 (7 parts), Functional safety of electrical/electronic/programmable electronic safety-related systems. IEC, 1998-2000.

[6] _ IEC61511, Functional safety - Safety instrumented systems for the process industry sector. IEC, 2004.

[7] ISA, Safety Instrumented Systems (SIS) - Safety Integrity Level (SIL), Evaluation Techniques (5 parts), ISA TR84.0.02. ISA, 2002.

[8] A. Villemeur, Reliability, availability, maintainability and safety assessment: methods and techniques., T. from French Edition by Cartier A. and L. M.C, Eds. Wiley, 1992.

[9] IEC, IEC 61025: Fault tree analysis. IEC, 1990. 
[10] J. Torres-Toledano and L. Sucar, "Bayesian networks for reliability of complex systems," in Progress in Artificial Intelligence, IBERAMIA98, H. Coelho, Ed. SpringerVerlag, 2004, pp. 195-206.

[11] Y. Dutuit, E. Chatelet, J.-P. Signoret, and P. Tomas, "Dependability modelling and evaluation by using stochastic petri nets: application to two test cases." Reliability Engineering and System Safety, vol. 55, pp. 117-124, 1997.

[12] S. Ferson, V. Kreinovich, L. Ginzburg, D. Myers, and K. Sentz, "Constructing probability boxes and dempstershafer structures," Tech. Rep., 2002.

[13] D. Ellsberg, "Risk, ambiguity and the savage's axioms," Quaterly Newspaper off Economics, vol. 75, pp. 643-669, 1961.

[14] P. Smets and R. Kennes, "The transferable belief model," Artificial Intelligence, vol. 66, pp. 191-243, 1994.

[15] R. Demirer and P. Shenoy, "Sequential valuation networks: A new graphical technique for asymmetric decision problem," Lecture Notes in Computer Science, pp. 252-265, 2001.

[16] F. Jensen, An Introduction to Bayesian Networks, U. Press, Ed., London, 1996.

[17] C. Huang and A. Dawiche, "Inference in belief networks: A procedural guide." International Journal of Approximate Reasoning, vol. 15, pp. 225-263, 1996.

[18] M. Valtorta, Y. Kim, and J. Vomlel, "Soft evidential update for probabilistic multiagent systems," International Journal of Approximate Reasoning, vol. 29, pp. 71-106, 2002.

[19] A. Bobbio, L. Portinale, M. Minichino, and E. Ciancamerla, "Improving the analysis of dependable systems by mapping fault trees into bayesian networks," Reliability Engineering and System Safety, vol. 71, no. 3, pp. 249260, March 2001.

[20] H. Boudali and J. Dugan, "A discrete-time bayesian network reliability modeling and analysis framework," Reliability Engineering and System Safety, vol. 87, pp. 337-349, 2005.

[21] A. Bobbio, E.Ciancamerla, G. Franceschinis, R. Gaeta, M. Minichino, and L. Portinale, "Sequential application of heterogeneous models for the safety analysis of a control system: a case study." Reliability Engineering and System Safety, vol. 81, pp. 269-280, 2003.

[22] A. Hoyland and M. Rausand, System reliability theory: models and statistical methods. Wiley, 1994.

[23] P. Weber and L. Jouffe, "Complex system reliability modelling with dynamic object oriented bayesian networks (doobn)." Reliability Engineering and System Safety, vol. 91, no. 2, pp. 149-162, February 2006.

[24] P. Shenoy, Valuation based systems: a framework for managing uncertainty in expert systems. Wiley, NewYork, 1992, pp. 83-104.

[25] P. Smets, TBMLAB, IRIDIA, Brussels, Belgium, March 2004.

[26] A. Dempster, "Upper and lower probabilities induced by a multivalued mapping," Annals of mathematical statistics, vol. 38, pp. 325-339, 1967.

[27] G. Shafer, A mathematical theory of evidence. Princeton University Press, 1976.

[28] P. Walley and S. Moral, "Upper probabilities based on the likelihood function," Journal of Royal Society B, vol. 61, no. 4, pp. 831-847, 1999.

[29] J. Kholas and P. Monney, A mathematical Theory of Hints: An approach to Dempster-Shafer Theory of evidence. Springer Verlag, 1995, vol. 425.

[30] R. Haenni and H. Lehmann, "Resource bounded and anytime approximation of belief function computation," International Journal of Approximate Reasoning, vol. 32, pp. 103-154, 2002.
[31] P. Smets, "The combination of evidence in the transferable belief model," IEEE Transactions on PAMI, vol. 12, no. 5, pp. 447-458, 1990.

[32] G. Klir and B. Yuan, Fuzzy Sets and Fuzzy Logic. Theory and Applications., U. S. River, Ed. NJ:Prentice Hall, 1995.

[33] M. Guth, "A probability foundation for vagueness and imprecision in fault tree analysis," IEEE Transactions on Reliability, vol. 40, no. 5, pp. 563-570, 1991.

[34] Y. Cheng, "Uncertainty in fault tree analysis," Tamkang Journal of Science and Engineering, vol. 3, no. 1, pp. 23$29,2000$.

[35] J. Hall and J. Lawry, "Imprecise probabilities of engineering system failure from random and fuzzy set reliability analysis," in ISIPTA'01, 26-29 june 2001.

[36] S. Ferson, Probability bounds analysis solves the problem of incomplete specification in probabilistic risk and safety assessments. American Society of Civil Engineers, Reston, Virginia, 2001, pp. 173-188.

[37] P. Walley, "Statistical reasoning with imprecise probabilities," 1991.

[38] P. Smets, "Decision making in the tbm: the necessity of the pignistic transformation," International Journal of Approximate Reasoning, vol. 38, pp. 133-147, 2005.

[39] - Constructing the pignistic probability function in a context of uncertainty, 1990, vol. 5, pp. 29-40.

[40] B. Cobb and P. Shenoy, "On the plausibility transformation method for translating belief function models to probability models," International Journal of Approximate Reasoning, 2005.

[41] S. Kamat and M. Riley, "Determination of reliability using event-based monte carlo simulation," IEEE Transactions on reliability, vol. 24, pp. 254-255, 1976.

Christophe SIMON was born in 1968. He received the MS in 1991 and the PHD in 1996. He is currently an assistant professor at the University of Nancy 2 since 1999. He is currently a researcher at the Resarch Centre for Automatic Control (CRAN) associated with the CNRS (UMR 7039). His area of interest are uncertainty in reliability modeling, system safety.

Philippe WEBER is an assistant professor at the University of Nancy 1 since 2001. He is currently a researcher at the Resarch Centre for Automatic Control (CRAN) associated with the CNRS (UMR 7039)

Eric LEVRAT Eric LEVRAT is an assistant professor at the University of Nancy 1 since 1990 . He is a researcher at the Resarch Centre for Automatic Control (CRAN) associated with the CNRS (UMR 7039). He focuses his interest on maintenance intelligent systems, prognosis and decision making processes, cost effectiveness maintenance policies. Since 2005 he is involved in an Integrated European Project DYNAMITE (Dynamic Decisions in Maintenance FP6-IST-NMP-2-017498). 